\title{
On the Classification of Control Sets
}

\author{
Fritz Colonius \\ Institut für Mathematik, Universität Augsburg, \\ 86135 Augsburg, Germany \\ and \\ Marco Spadini \\ Dipartimento di Matematica Applicata 'G. Sansone', \\ Università di Firenze, \\ Via S. Marta 3, 50139 Firenze, Italy
}

March 9, 2003

\begin{abstract}
The controllability behavior of nonlinear control systems is described by associating semigroups to locally maximal subsets of complete controllability, i.e., local control sets. Periodic trajectories are called equivalent if there is a 'homotopy' between them involving only trajectories. The resulting object is a semigroup, which we call the dynamic index of the local control set. It measures the different ways the system can go through the local control set.
\end{abstract}

\section{Introduction}

The purpose of this paper is to contribute to the qualitative study of the controllability behavior of nonlinear control systems. We classify the behavior within locally maximal subsets of complete controllability, local control sets as introduced in [4]. To each local control set a semigroup is associated which is constructed from periodic trajectories in the local control set. We take inspiration from the classical construction of the (first) homotopy group in algebraic topology, but use periodic orbits instead of closed loops. Two periodic orbits are equivalent, if they can be connected via a homotopy involving only periodic trajectories. This leads to some technical difficulties as it is necessary to make such an equivalence compatible with the natural composition of orbits with the same initial point. The resulting object is a commutative semigroup; in general it is not a group. This is due to the fact that for many nonlinear control systems the equivalence classes of periodic trajectories need not admit an inverse. We stress the fact that, even allowing trajectories followed backward in time, we 
would not in general obtain a group. In fact a neutral element would still be lacking.

The so-constructed semigroup is called dynamic index of the local control set. It measures the "different" ways in which the system can go through the local control set. It turns out, that for linear systems with controllable $(A, B)$ and admissible control range $U$ the index is always trivial. This remains true for small nonlinear perturbations. If the control range is small enough, we can also show that for a local control set around an attracting periodic solution of the uncontrolled system the index is isomorphic to the additive semigroup of natural numbers $\mathbb{N}$.

Compare also San Martin and Santana [11], where the homotopy type of Lie semigroups and invariant control sets is studied. We remark that in our construction the direction of the trajectories plays a crucial role. This is a decisive difference of our semigroup from homotopy groups. Katok and Hasselblatt [8, p. 117] briefly discuss other constructions of topological invariants using trajectories of dynamical systems. But perhaps closest in spirit to our paper are the papers $[12,13]$ by A. Sarychev. He studied homotopy properties of the space of trajectories. However, he was interested in the case, where the systems are completely controllable or, in our terminology, where the control set coincides with the whole state space. Furthermore, his main result concerns systems without drift where trajectories can be reversed.

After some basic definitions in Section 2 we define in Section 3 the key technical notion for the construction of the index, the so-called 'strong inner pairs', and show some of their relevant properties. Section 3 is devoted to the construction of the index and two simple examples are provided. Section 4 presents the explicit computation of the index in the case of the control set which arises, for a small control range, around an attracting periodic orbit of the uncontrolled system.

\section{Basic Definitions}

We will consider the following control-affine system in $\mathbb{R}^{d}$

$$
\begin{aligned}
\dot{x}(t) & =f(x(t), u(t)):=f_{0}(x(t))+\sum_{i=1}^{m} u_{i}(t) f_{i}(x(t)), \\
u & \in \mathcal{U}=\left\{u \in L_{\infty}\left(\mathbb{R}, \mathbb{R}^{m}\right), u(t) \in U \text { for almost all } t \in \mathbb{R}\right\} .
\end{aligned}
$$

with sufficiently smooth vector fields $f_{i}, i=0,1, \ldots, m$, on $\mathbb{R}^{d}$ and a compact convex neighborhood $U$ of the origin in $\mathbb{R}^{m}$. We assume that for every control $u \in \mathcal{U}$ and every initial condition $x(0)=x_{0} \in \mathbb{R}^{d}$ there exists a unique trajectory which we denote by $\varphi(t, x, u), t \in \mathbb{R}$. Our results will also hold-with some technical modifications-for systems on manifolds. Note that for control affine systems, the trajectories $\varphi(t, x, u)$ depend continuously on $(t, x, u)$, uniformly on bounded time intervals; here $\mathcal{U}$ will be considered in the weak ${ }^{*}$ topology inherited from the inclusion $\mathcal{U} \subset L_{\infty}\left(\mathbb{R}, \mathbb{R}^{m}\right)=\left(L_{1}\left(\mathbb{R}, \mathbb{R}^{m}\right)\right)^{*}$. Notice that $\mathcal{U}$ 
is in this topology a compact and separable metrizable space (see, e.g., Dunford/Schwartz [6]); an appropriate metric will be fixed throughout and denoted by 'd'; compare e.g. [1, Chapter 4] for this setting.

At some places we will consider for $0 \leq \rho \leq 1$, the control range $\rho U=\{\rho \cdot x$ : $x \in U\}$. Then we denote by $\mathcal{U}^{\rho}$ the set of all $L_{\infty}\left(\mathbb{R}, \mathbb{R}^{m}\right)$ control functions taking values in $\rho U$.

The following definitions specify subsets of complete approximate controllability, which are our primary concern in this paper.

Definition $1 A$ subset $D$ with nonempty interior of the state space $\mathbb{R}^{d}$ is a precontrol set if for all $x, y \in D$ and every $\varepsilon>0$ there exist $T>0$ and $u \in \mathcal{U}$ such that

$$
\varphi(t, x, u) \in D \text { for all } t \in[0, T] \quad \text { and } \quad|\varphi(T, x, u)-y|<\varepsilon \text {. }
$$

A precontrol set $D$ of $\mathbb{R}^{d}$ is a local control set if there exists a neighborhood $V$ of cl $D$ such that for every precontrol set $D^{\prime}$ with $D \subset D^{\prime} \subset V$ one has $D^{\prime}=D$.

Thus a local control set is a locally maximal precontrol set. Note also that control sets (with nonvoid interior) as discussed in [1] are globally maximal precontrol sets. The sets of reachable points from $x$ and controllable to $x \in \mathbb{R}^{d}$ in time $T>0$ are denoted by

$$
\mathcal{O}_{\leq T}^{+}(x)=\left\{y \in \mathbb{R}^{d}, \text { there are } 0 \leq t \leq T \text { and } u \in \mathcal{U} \text { with } y=\varphi(t, x, u)\right\}
$$

and

$$
\mathcal{O}_{\leq T}^{-}(x)=\left\{y \in \mathbb{R}^{d}, \text { there are } 0 \leq t \leq T \text { and } u \in \mathcal{U} \text { with } x=\varphi(t, y, u)\right\},
$$

respectively. Throughout this paper we require local accessibility, that is, $\mathcal{O}_{\leq T}^{+}(x)$ and $\mathcal{O}_{\leq T}^{-}(x)$ have nonvoid interiors for all $x \in \mathbb{R}^{d}$ and all $T>0$. Then in the interior of a local control set exact controllability holds. Recall also that local accessibility is guaranteed by the following accessibility rank condition:

$$
\operatorname{dim} \Delta_{\mathcal{L}}(x)=d \text { for all } x \in \mathbb{R}^{d},
$$

where $\mathcal{L}$ denotes the Lie algebra generated by the vector fields $f_{0}, \ldots, f_{m}$, and $\Delta_{\mathcal{L}}(x)$ is the subspace of the tangent space (identified with $\mathbb{R}^{d}$ ) generated by the vector fields in $\mathcal{L}$.

\section{Strong Inner Pairs}

In this section we specify the subclass of periodic trajectories which will be used for the construction of the dynamic index.

Definition 2 A pair $(u, x) \in \mathcal{U} \times \mathbb{R}^{d}$ is called a strong inner pair, if the control $u$ is piecewise constant with $u(t) \in \operatorname{int} U$ for all $t \in \mathbb{R}$ and there is $\delta>0$ such 
that for all $\tau>0$, small enough, and for all $y \in \mathbb{R}^{d}$ with $|x-y|<\delta$ the following property holds:

For all $0<t \leq \tau$ there are neighborhoods $N_{t}^{ \pm}(y)$ of $\varphi( \pm t, y, u)$ such that for any curve $\lambda \mapsto z_{\lambda}^{+} \in N_{t}^{+}(y)$ and $\lambda \mapsto z_{\lambda}^{-} \in N_{t}^{-}(y)$, with $z_{0}^{ \pm}=\varphi( \pm t, y, u)$, there are continuous maps

$$
\lambda \mapsto\left( \pm t_{\lambda}^{ \pm}, u_{\lambda}^{ \pm}\right):[0,1] \rightarrow(0, T) \times \mathcal{U},
$$

with $u_{\lambda}^{ \pm}$piecewise constant for $\lambda \in[0,1]$, and

$$
\left( \pm t_{0}^{ \pm}, u_{0}^{ \pm}\right)=( \pm t, u) \text { and } \varphi\left( \pm t_{\lambda}^{ \pm}, y, u_{\lambda}^{ \pm}\right)=z_{\lambda}^{ \pm} .
$$

The following remarks show that strong inner pairs are abundant provided that local accessibility holds. Here we write $e^{t X} x$, with $X=f(\cdot, u)$, in place of $\varphi(t, x, u)$ provided that $u$ is a constant control.

Remark 3 Assume that for some $\varepsilon>0$ there exist $s_{i}^{ \pm} \in(0, \varepsilon)$ and $u_{1}^{ \pm}, \ldots, u_{d}^{ \pm} \in$ int $U$ such that the two maps

$$
\left(t_{d}, \ldots, t_{1}\right) \mapsto e^{ \pm s_{d} X_{d}^{ \pm}} \cdots e^{ \pm s_{1} X_{1}^{ \pm}} x,
$$

where $X_{i}^{ \pm}:=f\left(\cdot, u_{i}^{ \pm}\right)$, have full rank on $(0, \varepsilon) \times \ldots \times(0, \varepsilon)$. On the interval $\left(-\sum_{i=1}^{d} s_{i}^{-}, \sum_{i=1}^{d} s_{i}^{+}\right]$, define

$$
u(t)= \begin{cases}u_{i}^{+} & \text {for } t \in\left(\sum_{j=1}^{i} s_{j}, \sum_{j=1}^{i+1} s_{j}\right], \\ u_{i}^{-} & \text {for } t \in\left(-\sum_{j=1}^{i+1} s_{j},-\sum_{j=1}^{i} s_{j}\right] .\end{cases}
$$

Then $(u, x)$ is a strong inner pair. To see that, it is sufficient to notice that the rank condition holds for any $y$ in a neighborhood of $x$ and that neighborhoods of $\varphi( \pm t, y, u)$ are of the form

$$
\left\{e^{ \pm t_{d} X_{d}} \ldots e^{ \pm t_{1} X_{1}} x \text {, with } t_{1}, \ldots, t_{d} \in(0, \varepsilon)\right\} .
$$

Hence the required continuous families can be obtained by appropriately changing the times $t_{i}$.

Strong inner pairs can also be easily obtained when the linearized control system is controllable. Here it is convenient-also for later use in Section 4-to consider $\rho$-dependent control ranges. Recall that for two vector fields $X, Y$ one defines $\operatorname{ad}_{X}^{0} Y=Y$ and for $k=1,2, \ldots$ one defines $\operatorname{ad}_{X}^{k} Y$ as the Lie bracket $\operatorname{ad}_{X}^{k} Y:=\left[X, \operatorname{ad}^{k-1} Y\right]$.

Proposition 4 Let $x \in \mathbb{R}^{d}$ and assume that

$$
\operatorname{span}\left\{\operatorname{ad}_{f_{0}}^{k} f_{i}(x), i=1, \ldots, m, k=0,1, \ldots\right\}=\mathbb{R}^{d} .
$$

Then for $\rho>0$, small enough, each $(u, y) \in \mathcal{U}^{\rho} \times \mathbb{R}^{d}$ with $u$ piecewise constant and $u \in \mathcal{U}^{\rho^{\prime}}$, for some $\rho^{\prime}<\rho$ and $|y-x|<\rho^{\prime}$, is a strong inner pair for the $\rho$-system. 
Sketch of the proof. If $\rho>0$ and $T>0$ are small enough, assumption (3) clearly holds for $\varphi(T, y, u)$ with $y$ in a small neighborhood of $x$ and $\|u\|_{\infty}<\rho$. This, for all $0<\tau \leq T$, guarantees controllability for the control system linearized along $\varphi(t, y, u)$ (with unbounded controls). Then a standard result in nonlinear control theory, see, e.g., [1, Theorem A.4.11 and Remark A.4.12] guarantees that the nonlinear control system with controls in $\mathcal{U}^{\rho}$ is locally controllable about the trajectory $\varphi(t, y, u)$, provided that $u \in \mathcal{U}^{\rho^{\prime}}$ for some $\rho^{\prime}<\rho$. This is based on an application of the inverse function theorem, which also provides the existence of neighborhoods $N_{t}^{ \pm}(y)$ as in Definition 2.

The following proposition shows, in particular, that the interior of a local control set corresponds to strong inner pairs.

Proposition 5 Let $D$ be a local control set for (1) and assume that the accessibility rank condition holds in $D$. Then, for any $x, y \in \operatorname{int} D$, there are $T>0$ and a T-periodic control function $u \in \mathcal{U}$ such that $(u, x)$ is a strong inner pair and $y \in \varphi([0, T], x, u)$.

Proof. By the accessibility rank condition, as in the proof of Krener's Theorem (compare [9]), it follows that there exist $u_{1}, \ldots, u_{d} \in \operatorname{int} U$ and $\delta>0$ such that,

$$
\begin{aligned}
& N^{+}=\operatorname{int}\left\{e^{t_{d} X_{d}} \cdots e^{t_{1} X_{1}} x: 0 \leq t_{i} \leq \delta, i=1, \ldots, d\right\} \neq \emptyset, \\
& N^{-}=\operatorname{int}\left\{e^{t_{d} X_{d}} \cdots e^{t_{1} X_{1}} x:-\delta \leq t_{i} \leq 0, i=1, \ldots, d\right\} \neq \emptyset
\end{aligned}
$$

where $X_{i}=f\left(\cdot, u_{i}\right)$ for $i=1, \ldots, d$.

Take $x^{+} \in N^{+}$. Since in the interior of $D$ approximate controllability holds, one can find a control function $v_{0}$ and a time $S_{0}$ such that $x^{-}:=\varphi\left(S_{0}, x^{+}, v_{0}\right) \in$ $N^{-}$. By continuous dependence we can assume that $v_{0}$ is a piecewise constant function with values in $\operatorname{int} U$. Let $v^{+}, v^{-} \in \mathcal{U}$ and $S^{+}, S^{-}>0$ be such that

$$
x^{+}=\varphi\left(S^{+}, x, v^{+}\right) \quad \text { and } \quad x=\varphi\left(S^{-}, x^{-}, v^{-}\right) .
$$

Concatenating $v^{-}, v^{+}$and $v_{0}$, and taking $T=S^{+}+S_{0}+S^{-}$one gets a $T$-periodic trajectory driven by some $T$-periodic piecewise constant control function $u$. One can also construct $u$ as a control function which connects $x^{+}$to $y$ and $y$ to $x^{-}$, in a way that essentially follows the line of the first part of the proof.

\section{The Dynamic Index}

In this section we construct a dynamic index for local control sets.

We consider a local control set $D$ for (1) and assume throughout that the accessibility rank condition holds. Define the set

$$
\mathcal{P}(D)=\left\{(T, u, x) \in(0, \infty) \times \mathcal{U} \times \mathbb{R}^{d}: \begin{array}{l}
(u, x) \text { is a } T \text {-periodic } \\
\text { strong inner pair, } T>0, \text { and } \\
\varphi(t, x, u) \in D, \forall t \in[0, T]
\end{array}\right\},
$$


endowed with the metric topology given by

$$
\operatorname{dist}((T, u, x),(S, v, y))=|T-S|+\|x-y\|_{\mathbb{R}^{d}}+\mathrm{d}(u, v) .
$$

Below, when no confusion can possibly arise, we shall omit the explicit dependence on the base set $D$.

Let us now introduce a relation on $\mathcal{P}$.

Definition $6(T, u, x) \sim(S, v, y)$ in $\mathcal{P}$ if there are $k+1$ elements $\left(T_{0}, u_{0}, x_{0}\right)$, $\ldots,\left(T_{k}, u_{k}, x_{k}\right)$ in $\mathcal{P}$ with the following properties:

(i) $\left(T_{0}, u_{0}, x_{0}\right)=(T, u, x)$ and $\left(T_{k}, u_{k}, x_{k}\right)=(S, v, y)$;

(ii) for $i=0, \ldots, k$ there are

$$
0=\tau_{i}^{0}<\ldots<\tau_{i}^{k_{i}}=T_{i} \text { and } 0=\sigma_{i+1}^{0}<\ldots<\sigma_{i+1}^{k_{i}}=T_{i+1},
$$

such that $\varphi\left(\tau_{i}^{j}, x_{i}, u_{i}\right)=x_{i}$ and $\varphi\left(\sigma_{i+1}^{j}, x_{i+1}, u_{i+1}\right)=x_{i+1}$ for all $i$ and all $j$;

(iii) there are continuous maps $H_{i}^{j}:[0,1] \rightarrow \mathcal{P}$ such that for $i=0, \ldots, k$ and $j=0, \ldots, k_{i}-1$

$$
\begin{aligned}
& H_{i}^{j}(0)=\left(\tau_{i}^{j+1}-\tau_{i}^{j}, u_{i}\left(\tau_{i}^{j}+\cdot\right), x_{i}\right), \quad \text { and } \\
& H_{i}^{j}(1)=\left(\sigma_{i+1}^{j+1}-\sigma_{i+1}^{j}, u_{i+1}\left(\sigma_{i+1}^{j}+\cdot\right), x_{i+1}\right) .
\end{aligned}
$$

In other words, $\left(T_{i}, u_{i}, x_{i}\right)$ and $\left(T_{i+1}, u_{i+1}, x_{i+1}\right)$ are chopped into $k_{i}$ periodic pieces of period $\tau_{i}^{j+1}-\tau_{i}^{j}$ and $\sigma_{i+1}^{j+1}-\sigma_{i+1}^{j}$ respectively, and the corresponding pieces are homotopic via trajectories.

This definition makes the natural operation of concatenation of trajectories 'compatible' with the relation ' $\sim$ '. To be more precise consider two continuous maps $H$ and $H^{\prime}$ from $[0,1]$ to $\mathcal{P}$. Clearly they establish 'homotopies' between $H(0)=(T, u, x)$ and $H(1)=(S, v, y)$, and between $H^{\prime}(0)=\left(T^{\prime}, u^{\prime}, x\right)$ and $H^{\prime}(1)=\left(S^{\prime}, v^{\prime}, y\right)$ respectively. Define $u \circ u^{\prime}$ as the concatenation on $\left[0, T+T^{\prime}\right]$ of $u$ and $u^{\prime}$ extended $\left(T+T^{\prime}\right)$-periodically to $\mathbb{R}$. According to Definition 6 $\left(T+T^{\prime}, u \circ u^{\prime}, x\right)$ is related to $\left(S+S^{\prime}, v \circ v^{\prime}, y\right)$; whereas there might not exist any continuous function $F:[0,1] \rightarrow \mathcal{P}$ with the property that $F(0)=(T+$ $\left.T^{\prime}, u \circ u^{\prime}, x\right)$ and $F(1)=\left(S+S^{\prime}, v \circ v^{\prime}, y\right)$.

Notice that the relation introduced above is an equivalence relation. Then, consider on $\mathcal{P} / \sim$, the set $\mathcal{Q}$ of all the formal (juxtaposition) products, i.e., the free semigroup on $\mathcal{P} / \sim$. (See, e.g., Howie [7] for some general facts about the algebraic theory of semigroups.) Usually, we shall set

$$
[T, u, x]^{n}=\underbrace{[T, u, x] \cdots[T, u, x]}_{n \text { times }},
$$

for any $n \geq 0$. Here the square parentheses denote the equivalence classes. 
Clearly $\mathcal{Q}$ is a semigroup which, besides its non-commutativity, is far too large for being of any use. Below we factorize it over the congruence induced by two families of equations among the elements of $\mathcal{Q}$. Recall that a congruence on a semigroup $(S, \cdot)$ is an equivalence relation ' $\equiv$ ' such that

$$
a \equiv a^{\prime} \text { and } b \equiv b^{\prime} \text { imply } a \cdot b \equiv a^{\prime} \cdot b^{\prime},
$$

for any $a, a^{\prime}, b, b^{\prime} \in S$.

Consider the following families of relations:

$$
\begin{aligned}
\mathcal{F} & =\{[T, u, x][S, v, x]=[T+S, u \circ v, x]:(T, u, x),(S, v, x) \in \mathcal{P}\}, \\
\mathcal{G} & =\{[T, u, x][S, v, y]=[S, v, y][T, u, x]:(T, u, x),(S, v, y) \in \mathcal{P}\} .
\end{aligned}
$$

Notice that the elements of $\mathcal{F}$ are well defined. In fact, by the definition of ' $\sim$ ' one has that

$$
(T, u, x) \sim(\bar{T}, \bar{u}, \bar{x}) \text { and }(S, v, x) \sim(\bar{S}, \bar{v}, \bar{x})
$$

imply

$$
(T+S, u \circ v, x) \sim(\bar{T}+\bar{S}, \bar{u} \circ \bar{v}, \bar{x}) .
$$

The union of the families $\mathcal{F}$ and $\mathcal{G}$ clearly can be seen as a relation on $\mathcal{Q}$, i.e., as a subset of $\mathcal{Q} \times \mathcal{Q}$. Now, since the intersection of congruences is again a congruence, it makes sense to consider the congruence $(\mathcal{F} \cup \mathcal{G})^{\#}$ generated by the set $\mathcal{F} \cup \mathcal{G}$, namely the intersection of all the congruences containing $\mathcal{F} \cup \mathcal{G}$ (see e.g. [7]).

Finally, we define the dynamic index $\mathcal{I}(D)$ of $D$ as the quotient

$$
\mathcal{I}(D):=\mathcal{Q}(D) /(\mathcal{F} \cup \mathcal{G})^{\#}
$$

Notice that $\mathcal{I}(D)$ is a commutative semigroup. Next we consider two easy examples.

Example 7 (Linear Systems) Consider the following linear control system with restricted control range

$$
\dot{x}(t)=A x(t)+B u(t) \quad \text { in } \mathbb{R}^{d}, u \in \mathcal{U},
$$

where $U \subset \mathbb{R}^{m}$ is convex and compact with $0 \in \operatorname{int} U$ and $A$ and $B$ are constant matrices of dimensions $d \times d$ and $d \times m$, respectively. We assume that the pair $(A, B)$ is controllable, i.e., that rank $\left[B, A B, \ldots A^{d-1} B\right]=d$. Then the index $\mathcal{I}(D)$ of the unique control set $D$ reduces to the unity. In fact: For a T-periodic strong inner pair $(u, x)$ in the interior of $D$, define a homotopy to the origin via

$$
H(\alpha):=(T, \alpha u, \alpha x), \alpha \in[0,1] .
$$

Linearity implies that $\varphi(T, \alpha x, \alpha u)=\alpha x$ for all $\alpha \in[0,1]$. Hence this is a periodic solution, and for $\alpha=0$ one obtains the equilibrium. If $0 \notin U$ then the same result holds provided that $A$ is hyperbolic (cp. [3]). 
Example 8 (Small perturbations of linear systems) Consider a control process of the form:

$$
\dot{x}(t)=A x(t)+B u(t)+\sum_{i=1}^{m} u_{i}(t) F_{i}(x(t)), u \in \mathcal{U},
$$

with $U \subset \mathbb{R}^{m}$ compact and convex with non empty interior. Assume that $(A, B)$ in (4) is controllable and $A$ is hyperbolic, and let $F_{i}$ be $C^{1}$.

It follows from the proof of the uniqueness for perturbations in [4] that there exists $M>0$ such that, if

$$
\left\|D_{1} F_{i}(x, u)\right\| \leq M, \quad \text { and } \quad\left\|D_{2} F_{i}(x, u)\right\| \leq M
$$

for all $(x, u)$ and $i=1, \ldots, m$, then the control process (4) admits exactly one control set $D$. Furthermore, the dynamic index $\mathcal{I}(D)$ reduces to its unity. In fact, in [4], we constructed a homotopy between any two given periodic triples (possibly with different periods).

\section{The index of a control set near a periodic orbit}

This section is devoted to the computation of the index of the control set for (1) which arise for a small control range around an isolated attracting periodic orbit $\gamma=\varphi\left([0, T], x_{0}, 0\right)$, with (minimal) period $T>0$, of the uncontrolled system, assuming that the linearized system along $\gamma$ is controllable. Recall that a periodic orbit (of an autonomous differential equation) is called attracting, if the eigenvalues of the linearized Poincaré map are strictly smaller than one in modulus; compare [10].

Proposition 9 Let $\gamma$ be a attracting orbit of the uncontrolled system, and let $A$ be a neighborhood of $\gamma$. Assume that the controllability rank condition (3) holds. Then there exist $\rho_{0}$ such that for any $0<\rho \leq \rho_{0}$ there exists a unique control set $D^{\rho}$ with $\gamma \subset D^{\rho} \subset A$.

Proof. The controllability rank condition implies by Proposition 4 that all pairs $(x, 0) \in \gamma \times \mathcal{U}^{\rho}$ are strong inner pairs, hence inner pairs. Then Corollary 4.7.6 in [1] implies the assertion.

We shall prove that, when $\rho$ is small enough, the index of the control set $D^{\rho}$ containing the $T$-periodic orbit $\gamma$ is isomorphic to $\mathbb{N}$. To prove this result we need to show that when $\left(T_{1}, u_{1}, x_{1}\right) \in \mathcal{P}\left(D^{\rho}\right)$ is such that $\varphi\left(\left[0, T_{1}\right], x_{1}, u_{1}\right)$ goes $n$ times around $\gamma$, then $\left(T_{1}, u_{1}, x_{1}\right) \sim\left(n T, 0, x_{0}\right)$ and therefore $\left[T_{1}, u_{1}, x_{1}\right]=$ $\left[T, 0, x_{0}\right]^{n}$. To make this precise we shall introduce Definition 11 below.

However, it is first necessary to establish some preliminaries on the Poincaré map for control systems. We will use some notions and results from Colonius/Sieveking [2].

Definition 10 Let $x_{0} \in \mathbb{R}^{n}, L: \mathbb{R}^{d} \rightarrow \mathbb{R}$ linear and $\alpha>0$. If $L f(x, u)>\alpha$ for all $x$ in a neighborhood $W$ of $x_{0}$ and all $u \in U$ then the connected component of $W \cap L^{-1}\left(L x_{0}\right)$ containing $x_{0}$ is called a local transversal section through $x_{0}$. 

side".

Obviously, trajectories "can cross a local transversal section only from one

Definition 11 Let $\Omega$ be a neighborhood of $\gamma$. We say that a closed orbit $\gamma_{1}=$ $\varphi\left(\left[0, T_{1}\right], x_{1}, u_{1}\right) \subset \Omega$ goes $n$ times around $\gamma$ (relatively to $\Omega$ ) if there exists a linear map $L$ as in Definition 10 such that

1. $S:=\Omega \cap L^{-1}\left(L x_{0}\right)$ is a local transversal section to $\gamma$,

2. $\gamma \cap S=\left\{x_{0}\right\}$,

3. $x_{1} \in S$, and

4. there exist exactly $n$ times $t_{i} \in\left(0, T_{1}\right], i=1, \ldots, n$, such that $\varphi\left(t_{i}, x_{1}, u_{1}\right) \in$ $S$.

It is a consequence of the Hahn-Banach Theorem that $x_{0}$ admits a local transversal section if $0 \notin f\left(x_{0}, U\right)$, since the set $f\left(x_{0}, U\right)$ is convex and compact. Therefore, if $x_{0}$ is not an equilibrium of the uncontrolled system, i.e., if $0 \neq$ $f\left(x_{0}, 0\right)$, then $x_{0}$ admits a local transversal section for the system with control range $\rho U$ with $\rho$ small enough.

Definition 12 Let $S$ be a local transversal section through $x_{0}$, and let $V_{1} \subset V_{0}$ be neighborhoods of $x_{0}$. The triple $\left(V_{0}, V_{1}, S\right)$ is a flow box around $x_{0}$ if it has the following property:

If $\varphi\left(\cdot, x_{0}, u\right)$ satisfies

$$
\varphi\left(t_{0}, x_{0}, u\right) \notin V_{0}, \quad \varphi\left(t_{1}, x_{0}, u\right) \in V_{1}, \quad \varphi\left(t_{2}, x_{0}, u\right) \notin V_{0}
$$

for some $0 \leq t_{0}<t_{1}<t_{2}$, then there exists $t \in\left(t_{0}, t_{2}\right)$ such that $\varphi\left(t, x_{0}, u\right) \in S$ and $\varphi\left(s, x_{0}, u\right) \in V_{0}$ for all $s$ between $t$ and $t_{1}$.

The following result establishes the existence of flow boxes; it is a slight modification of Theorem 2.16 in [2].

Lemma 13 Let $S$ be a local transversal section through $x_{0}$. Then for every neighborhood $W$ of $S$ there are neighborhoods $V_{0}$ and $V_{1}$ of $x_{0}$ contained in $W$ such that $\left(V_{0}, V_{1}, S\right)$ is a flow box around $x_{0}$.

Proof. There exist a linear map $L: \mathbb{R}^{d} \rightarrow \mathbb{R}$, a constant $\alpha>0$, and a neighborhood $W_{1} \subset W$ of $x_{0}$ with $S \supset W_{1} \cap L^{-1}\left(L x_{0}\right)$ and

$$
L f(y, v)>\alpha \text { for all } y \in W_{1}, v \in U .
$$

Choose a ball $V_{0}=B\left(r_{0}, x_{0}\right)$ around $x_{0}$ with radius $r_{0}>0$ such that $V_{0} \subset W_{1}$ and set $c:=\sup \{|f(y, u)|, y \in V$ and $v \in U\}$. Then choose $r_{1} \in\left(0, r_{0}\right)$ so small that

$$
L z-\alpha / 2 c\left(r_{0}-r_{1}\right) \leq L y \leq L z+\alpha / 2 c\left(r_{0}-r_{1}\right)
$$


for all $z, y \in V_{1}=B\left(r_{1}, x_{0}\right)$. We have for $t>t^{\prime} \geq 0$ :

$$
\varphi(t, x, u)=\varphi\left(t^{\prime}, x, u\right)+\int_{t^{\prime}}^{t} f(\varphi(s, x, u), u(s)) d s
$$

and hence

$$
\begin{aligned}
L \varphi(t, x, u) & =L \varphi\left(t^{\prime}, x, u\right)+\int_{t^{\prime}}^{t} L f(\varphi(s, x, u), u(s)) d s \\
& \geq L \varphi\left(t^{\prime}, x, u\right)+\alpha\left(t-t^{\prime}\right),
\end{aligned}
$$

provided that $\varphi(s, x, u) \in W_{1}, t^{\prime} \leq s \leq t$. Without loss of generality, we may assume

$$
\varphi(s, x, u) \in V_{0} \text { for all } t_{0} \leq s \leq t_{2}
$$

replacing, if necessary, $t_{0}$ by the last time before $t_{1}$ at which $\varphi(t, x, u)$ is in the complement of $V_{0}$ and $t_{2}$ by the first time after $t_{1}$ at which $\varphi(t, x, u)$ leaves $V_{0}$. We have

$$
\begin{aligned}
& r_{0}-r_{1} \leq\left|\varphi\left(t_{1}, x, u\right)-\varphi\left(t_{0}, x, u\right)\right| \leq c\left(t_{1}-t_{0}\right), \\
& r_{0}-r_{1} \leq\left|\varphi\left(t_{2}, x, u\right)-\varphi\left(t_{1}, x, u\right)\right| \leq c\left(t_{2}-t_{1}\right) .
\end{aligned}
$$

If $L \varphi\left(t_{0}, x, u\right) \leq L x_{0} \leq L \varphi\left(t_{2}, x, u\right)$, or $L \varphi\left(t_{2}, x, u\right) \leq L x_{0} \leq L \varphi\left(t_{1}, x, u\right)$, the assertion follows by continuity of $t \mapsto L f(t, x, u)$. Hence we only have to consider the following two cases:

1. $L x_{0}<\min \left\{L \varphi\left(t_{0}, x, u\right), L \varphi\left(t_{2}, x, u\right)\right\}$. Here $L \varphi\left(t_{1}, x, u\right) \geq L \varphi\left(t_{0}, x, u\right)+$ $\alpha\left(t_{1}-t_{0}\right)>L x_{0}+\alpha / c\left(r_{0}-r_{1}\right)$, contradicting (5) for $y=L \varphi\left(t_{1}, x, u\right)$.

2. $L x_{0}>\max \left\{L \varphi\left(t_{0}, x, u\right), L \varphi\left(t_{2}, x, u\right)\right\}$. Here $L \varphi\left(t_{2}, x, u\right) \geq L \varphi\left(t_{1}, x, u\right)+$ $\alpha\left(t_{2}-t_{1}\right)>L \varphi\left(t_{1}, x, u\right)+\alpha / c\left(r_{0}-r_{1}\right)$, again contradicting (5).

We now turn to the Poincaré map. Note that for $\rho=0$ a local transversal section as in Definition 10 coincides with the usual notion of a local transversal section for autonomous differential equations. Then it defines for $\rho>0$, small enough, also a local transversal section for the system with control range $\rho U$.

Proposition 14 Let $S$ be a local transversal section through $x_{0} \in \gamma$. If $\rho$ is small enough, there exists a neighborhood $V$ of $x_{0}$ in $S$ such that the Poincaré first return map $P: V \times \mathcal{U}^{\rho} \rightarrow S$ is well-defined and continuous. Moreover, the map that takes $(x, u)$ into the 'first return time' $\tau(x, u)$ is continuous.

Proof. Let us first show that $P$ is well-defined. Notice that the orbits can cross $S$ only from one side; therefore it is sufficient to show that there exists a neighborhood $V \subset S$ of $x_{0}$ such that the orbits return to $S$ after a finite time.

Let $W$ be a neighborhood of $x_{0}$ in $\mathbb{R}^{d}$ and $\left(V_{0}, V_{1}, S\right)$ be a flow box around $x_{0}$ with $\mathrm{cl} V_{0} \subset W$. Taking if necessary a smaller $W$, we can assume that there 
are times $t_{0}$ and $t_{1}$, with $0<t_{0}<T<t_{1}$, for which $\varphi\left(t_{0}, x_{0}, 0\right)$ and $\varphi\left(t_{1}, x_{0}, 0\right)$ are in $W \backslash \mathrm{cl} V_{0}$.

By continuous dependence on initial data there exist a neighborhood $V \subset V_{1}$ of $x_{0}$ in $S$ and $\rho_{0}>0$ such that, for $0<\rho<\rho_{0}$ and for every $(x, u) \in V \times \mathcal{U}^{\rho}$

$$
\varphi\left(t_{0}, x, u\right) \in W \backslash \operatorname{cl} V_{0}, \varphi\left(t_{1}, x, u\right) \in W \backslash \operatorname{cl} V_{0}, \varphi(T, x, u) \in V_{1} .
$$

Since $\left(V_{0}, V_{1}, S\right)$ is a flow box, for each $(x, u) \in V \times \mathcal{U}^{\rho}$ there exists a time $\tau(x, u)$, with $t_{0}<\tau(x, u)<t_{1}$ such that $\varphi(\tau(x, u), x, u) \in S$. For $W$ small enough this time is unique proving that $P(x, u):=\varphi(\tau(x, u), x, u)$ is well-defined.

We shall now prove continuity of the map $(x, u) \mapsto P(x, u)$. Consider a sequence $\left\{\left(\xi_{n}, u_{n}\right)\right\}$ in $S \times \mathcal{U}^{\rho}$ converging to $\left(\xi_{0}, u_{0}\right)$. Fix a neighborhood $W$ of $P\left(\xi_{0}, u_{0}\right)$ in $S$ and let $\hat{W}$ be a neighborhood of $P\left(\xi_{0}, u_{0}\right)$ in $\mathbb{R}^{d}$ such that $W=\hat{W} \cap S$. Let $\left(V_{0}, V_{1}, S\right)$ be a flow box around $P\left(\xi_{0}, u_{0}\right)$ with $\operatorname{cl} V_{0} \subset \hat{W}$.

Let $\tau=\tau\left(\xi_{0}, u_{0}\right)$. As in the first part of the proof, taking $W$ smaller if necessary, one can find times $0<\tau_{0}<\tau<\tau_{1}$ such that

$$
\varphi\left(\tau_{0}, x_{0}, u_{0}\right), \varphi\left(\tau_{1}, x_{0}, u_{0}\right) \in W \backslash \operatorname{cl} V_{1} .
$$

From [1, Lemma 4.3.2] one has

$$
\begin{aligned}
\lim _{n \rightarrow \infty} \varphi\left(\tau, \xi_{n}, u_{n}\right) & =\varphi\left(\tau, \xi_{0}, u_{0}\right)=P\left(\xi_{0}, u_{0}\right), \\
\lim _{n \rightarrow \infty} \varphi\left(\tau_{0}, \xi_{n}, u_{n}\right) & =\varphi\left(\tau_{0}, \xi_{0}, u_{0}\right), \\
\lim _{n \rightarrow \infty} \varphi\left(\tau_{1}, \xi_{n}, u_{n}\right) & =\varphi\left(\tau_{1}, \xi_{0}, u_{0}\right) .
\end{aligned}
$$

Therefore, for $n$ large enough,

$$
\varphi\left(\tau_{0}, x_{n}, u_{n}\right), \varphi\left(\tau_{1}, x_{n}, u_{n}\right) \notin V_{0} \quad \text { and } \quad \varphi\left(\tau, x_{n}, u_{n}\right) \in V_{1} .
$$

Since $\left(V_{0}, V_{1}, S\right)$ is a flow box there exists $\tau_{n} \in\left(\tau_{0}, \tau_{1}\right)$ such that $P\left(x_{n}, u_{n}\right)=$ $\varphi\left(\tau_{n}, x_{n}, u_{n}\right) \in S \cap W$. This proves that, for $n$ large, $P\left(x_{n}, u_{n}\right) \in W$ and continuity follows. Notice also that, in the construction above, $\tau_{n}=\tau\left(\xi_{n}, u_{n}\right)$ satisfies

$$
\tau_{1}-\tau_{0}>\left|\tau-\tau_{n}\right|
$$

by shrinking $W$, we can make the differences $\tau_{1}-\tau_{0}$ as small as we please, therefore proving the continuity of the map $(x, u) \mapsto \tau(x, u)$.

The (technically involved) proof of the following proposition is given in [5]. Here for a control function $u$ and a time $T>0$ the function $u \mid[0, T]$ extended periodically to $\mathbb{R}$ is denoted by $[u]_{T}$.

Proposition 15 Let $\lambda \mapsto T_{\lambda}:[0,1] \rightarrow \mathbb{R}$ be continuous. Then, for a (fixed) control function $u \in \mathcal{U}$, the map $\lambda \mapsto u_{\lambda}:=\lambda[u]_{T_{\lambda}}:[0,1] \rightarrow \mathcal{U}$ is continuous.

The next fact is crucial for the construction of the homotopy between the orbits that wind $n$ times around $\gamma$ and $[T, 0, x]^{n}$. 
Lemma 16 Assume that the T-periodic orbit $\gamma=\varphi\left([0, T], x_{0}, 0\right)$ is attracting, and let $S$ be a local transversal section for the orbit $\gamma$ through $x_{0}$. Then there exists $\rho>0$ and a neighborhood $V$ of $x_{0}$ such that $P(\cdot, u)$ is a contraction, uniformly for $u \in \mathcal{U}^{\rho}$.

Proof. Without loss of generality we can assume that $S$ lies on the hyperplane $X:=\left\{x^{d}=0\right\} \subset \mathbb{R}^{d}$. Here and along all this proof the exponent $d$ denotes the $d$-th component in $\mathbb{R}^{d}$. Take

$$
\mathcal{F}:=\mathcal{U}^{\rho} \cap C^{1}\left(\mathbb{R}, \mathbb{R}^{m}\right)
$$

with the $L_{\infty}$ topology, and define for $u \in \mathcal{F}$ the $C^{1}$ function $\Psi_{u}: \mathbb{R} \times X \rightarrow \mathbb{R}$ as

$$
\Psi_{u}(t, y):=\varphi^{d}\left(t+\tau_{u}, y, u\right)-y^{d},
$$

where $\tau_{u}=\tau\left(x_{0}, u\right)$. One has $\Psi_{u}\left(0, x_{0}\right)=0$. Observe that $\Psi_{u}$ is continuous, uniformly for $u \in \mathcal{F}$. Moreover, reducing $\rho$ if necessary, one can find $\delta>0$ such that

$$
D_{1} \Psi_{u}\left(0, x_{0}\right)=f^{d}\left(\varphi\left(\tau_{u}, x_{0}, u\right), u\left(\tau_{u}\right)\right) \geq 1 / \delta .
$$

A parametrized version of the implicit function theorem implies the existence of a neighborhood $V$ of $x_{0}$ in $S$ and of a $C^{1}$ function $t_{u}: V \rightarrow \mathbb{R}$ such that $\Psi_{u}\left(t_{u}(x), x\right)=0$ for every $x \in V$.

Clearly, if $\rho$ is small enough and $x \in V$, then the time $\tau(x, u)$ for the Poincaré map coincides with $t_{u}(x)+\tau_{u}$. Thus $D_{1} \tau(x, u)$ is well defined and the map

$$
(x, u) \mapsto D_{1} P(x, u)=D_{1} \varphi(\tau(x, u), x, u) D_{1} \tau(x, u)+D_{2} \varphi(\tau(x, u), x, u)
$$

is continuous. Since $\gamma$ is attracting, the eigenvalues of $D_{1} P\left(x_{0}, 0\right)$ are strictly smaller than one in modulus. Thus there exists a norm on $S$ such that the operator $D_{1} P\left(x_{0}, 0\right)$ has norm smaller than one. By continuity and restricting $V$ and $\rho$ if necessary, we can assume that the same is true for $D_{1} P(x, u)$ for every $x \in V$ and $u \in \mathcal{U}^{\rho} \cap C^{1}\left(\mathbb{R}, \mathbb{R}^{m}\right)$. Whence it follows that $P(\cdot, u)$ is a contraction with constant

$$
k=\sup _{(\xi, \mu) \in V \times \mathcal{U}^{\rho}}\left\|D_{1} P(\xi, \mu)\right\|<1 .
$$

Let us show that $P(\cdot, u)$ remains a $k$-contraction when $u$ is a general (not necessarily continuously differentiable) element of $\mathcal{U}^{\rho}$. Since the $C^{1}$ functions are dense in $\mathcal{U}^{\rho}$ in the weak topology, there is a sequence $\left\{u_{n}\right\}$ of $C^{1}$ functions in $\mathcal{U}^{\rho}$ converging to $u_{0}$ in the weak* topology. Take $x$ and $y$ in $V$, by Proposition 14 we know that $P$ is continuous when $\mathcal{U}^{\rho}$ is endowed with the weak* topology. Therefore, for $\varepsilon>0$, one has

$$
\left|P(x, u)-P\left(x, u_{n}\right)\right|+\left|P(y, u)-P\left(y, u_{n}\right)\right|<\varepsilon,
$$

for $n$ sufficiently large. Therefore

$$
\begin{aligned}
|P(x, u)-P(y, u)| \leq & \left|P(x, u)-P\left(x, u_{n}\right)\right|+\left|P\left(x, u_{n}\right)-P\left(y, u_{n}\right)\right| \\
& +\left|P\left(y, u_{n}\right)-P(y, u)\right| \\
\leq & k|x-y|+\varepsilon .
\end{aligned}
$$


Since $\varepsilon>0$ is arbitrary, this proves the assertion.

Proposition 17 Assume that the T-periodic orbit $\gamma=\varphi\left([0, T], x_{0}, 0\right)$ is attracting, and let $S$ be a local transversal section for $\gamma$ through $x_{0}$. Then there exists $\rho>0$ and a neighborhood $V$ of $x_{0}$ such that for every $n \in \mathbb{N}$ and every $(x, u) \in V \times \mathcal{U}^{\rho}$ the map $(x, u) \rightarrow P^{n}(x, u)$ is well defined.

Moreover, for every $u \in \mathcal{U}^{\rho}$, there exists a $T_{u}>0$ and a unique $T_{u}$-periodic solution $\varphi\left(\cdot, x_{u},[u]_{T_{u}}\right)$ winding $n$ times around $\gamma$, and the functions $u \mapsto T_{u}$ and $u \mapsto x_{u}$ are continuous.

Proof. In Lemma 16 we proved that for $u \in \mathcal{U}^{\rho}$, with $\rho$ sufficiently small, $P(\cdot, u)$ is a contraction on $\mathrm{cl} V$. Consequently, we can assume that $P(\operatorname{cl} V, u) \subset$ cl $V$ for any $u \in \mathcal{U}^{\rho}$. In particular $P^{n}(\cdot, u)$ is well-defined for any $n \in \mathbb{N}$ and $u \in \mathcal{U}^{\rho}$. Notice also that for every $n \in \mathbb{N}$ and $u \in \mathcal{U}^{\rho}$ also $P^{n}(\cdot, u)$ is a contraction. Therefore, given $n$ and $u$ as above, there exist a unique fixed point $x_{u}$ for $P^{n}(\cdot, u)$ in $N$ which depends continuously on $u$. Define $T_{u}$ as the time needed for $\varphi\left(\cdot, x_{u}, u\right)$ to reach $x_{u}$ after winding $n$ times around $\gamma$. Continuous dependence of $T_{u}$ on $u$ follows from continuity of the first return time as shown in Proposition 14.

Notice that in $P^{n}(x, u)$ the control $u$ restricted to $\left[0, T_{u}\right]$ is applied $n$ times. When the control function is identically zero and $V$ is small enough, the sets of fixed points of $P(\cdot, 0)$ and of $P^{n}(\cdot, 0)$ reduce to $x_{0}$. However, this needs not be true when nontrivial controls are applied. Indeed, when the controllability condition (3) is satisfied one can for $n>1$ construct a (small) control $u$ which yields a periodic trajectory meeting the transversal section in $n$ distinct points. (This can be deduced from Proposition 9.) Thus, for such $u$, the fixed point set of $P^{n}(\cdot, u)$ strictly contains that of $P(\cdot, u)$.

We are finally in a position to prove the claim we made at the beginning of this section.

Theorem 18 Assume that the uncontrolled system has an attracting T-periodic solution $\varphi\left(\cdot, x_{0}, 0\right)$ with $T>0$, and that the controllability condition (3) is satisfied. Then, when $\rho$ is small enough, the dynamic index $\mathcal{I}\left(D^{\rho}\right)$ of the control set $D^{\rho}$ containing $\gamma:=\varphi\left([0, T], x_{0}, 0\right)$ is isomorphic to $\mathbb{N}$.

Proof. Let $N=\operatorname{cl} V$ be the compact neighborhood of $x_{0}$ found in the proof of Proposition 17 above. Consider a $T_{1}$-periodic orbit $\varphi(\cdot, x, u)$ with $x \in N$, $u \in \mathcal{U}^{\rho^{\prime}}$ for some $0<\rho^{\prime}<\rho$ and $u$ piecewise constant. There exists $n$ such that $\varphi\left(T_{1}, x, u\right)=P^{n}(x, u)$. By Proposition 17, there exist $T_{\lambda}>0$ and a unique $T_{\lambda}$-periodic solution $\varphi\left(\cdot, x_{\lambda},[\lambda u]_{T_{\lambda}}\right)$ winding $n$ times around $\gamma$. By Proposition 15 the map $\lambda \mapsto u_{\lambda}:=[\lambda u]_{T_{\lambda}}$ is continuous. Hence, again by Proposition 17, it follows that $T_{\lambda}$ and $x_{\lambda}$ depend continuously on $\lambda$. In particular, $T_{0}=n T$. Since by Proposition $4\left([v]_{T_{\lambda}}, x_{\lambda}\right)$ is a strong inner pair for each $\lambda$, this yields the desired homotopy between $\left(T_{1}, u_{1}, x_{1}\right)$ and $\left(T_{0}, 0, x_{0}\right)$.

We conclude the paper with a remark showing that the dynamic index allows us to distinguish control sets around an attracting periodic orbit as above from control sets around a homoclinic orbit. 
Remark 19 Suppose that the uncontrolled system has a homoclinic orbit given by

$$
\varphi\left(t, x_{1}, u_{1}\right), t \in \mathbb{R}, \text { with } \lim _{t \rightarrow \pm \infty} \varphi\left(t, x_{1}, u_{1}\right)=x_{0},
$$

where $x_{0}$ is an equilibrium of the uncontrolled system. If the controllability condition (3) holds for all points in $\gamma:=\left\{x_{0}\right\} \cup\left\{\varphi\left(t, x_{1}, u_{1}\right), t \in \mathbb{R}\right\}$ and this is a chain recurrent component of the uncontrolled system, then for every $\rho>0$ there is a control set $D^{\rho}$ containing this set in its interior and

$$
\bigcap_{\rho>0} D^{\rho}=\gamma
$$

see Corollary 4.7.6 in [1]. For any small $\rho$, the index $\mathcal{I}\left(D^{\rho}\right)$ contains an element $\left[T, x_{0}, 0\right]$ which is idempotent, i.e., $\left[T, x_{0}, 0\right]^{2}=\left[T, x_{0}, 0\right]$. Hence $\mathcal{I}\left(D^{\rho}\right)$ is not isomorphic to $\mathbb{N}$.

\section{References}

[1] F. Colonius and W. Kliemann, The Dynamics of Control, Birkhäuser, Boston, 2000.

[2] F. Colonius and M. Sieveking, Asymptotic properties of optimal solutions in planar discounted control problems, SIAM J. Control and Optimization, 27 (1989), 608-630.

[3] F. Colonius and M. Spadini, Uniqueness of Control Sets for Perturbations of Linear Systems, Stability and Stabilization of Nonlinear Systems, D. Aeyels, F. Lamnabhi-Lagarrigue and A. van der Schaft, eds., Lecture Notes in Control and Information Sciences, 246, Springer Verlag, 1999.

[4] F. Colonius and M. Spadini, Local control sets, submitted.

[5] F. Colonius and M. Spadini, A dynamic index for control sets, submitted.

[6] N. Dunford and J. T. Schwartz, Linear Operators, Part I: General Theory, Wiley-Interscience, 1977.

[7] J. M. Howie An Introduction to Semigroups Theory, Academic Press, London, 1976.

[8] A. Katok and B. Hasselblatt, Introduction to the Modern Theory of Dynamical Systems, Cambridge University Press, Cambridge, 1995.

[9] A. J. Krener, A generalization of Chow's theorem and Bang-Bang theorem for nonlinear control problems, SIAM J. Control Optim., 14 (1974), 43-52.

[10] C. Robinson, Dynamical Systems: Stability, Symbolic Dynamics, and Chaos, CRC Press, 1995. 
[11] L.A. San Martin and A.J. Santana, The homotopy type of Lie semigroups in semisimple Lie groups, Instituto de Matematica, Universidade Estadual de Campinas, Brasil, 1999.

[12] A.V. Sarychev, On topological properties of trajectory space for nonlinear control systems, Analysis of Nonlinear Controlled Dynamical Systems, B. Bride, B. Bonnard, G.P. Gauthier, I. Kupka, eds., Birkhäuser, pp. 358-371, 1991.

[13] A.V. Sarychev, On homotopy properties of trajectories for completely nonholonomic differential systems, Doklady Akad. Nauk SSSR Vol. 314, pp. 1336-1340, 1990. English translation Doklady Vol. 42, pp. 674-678, 1991. 